Published in final edited form as:

Epidemiology. 2017 November ; 28(6): e62-e63. doi:10.1097/EDE.0000000000000707.

\title{
The Authors Respond
}

\author{
Dr. SR Cole, \\ Department of Epidemiology, UNC-Chapel Hill \\ Dr. H Chu, \\ Department of Biostatistics, University of Minnesota \\ Dr. MA Brookhart, and \\ Department of Epidemiology, UNC-Chapel Hill \\ Dr. JK Edwards \\ Department of Epidemiology, UNC-Chapel Hill
}

\section{To the Editor}

In darkness of night without lumen, one takes voices heard to be human.

We thank Dr. McIsaac ${ }^{1}$ for his interest in our letter. ${ }^{2}$ We apologize that in our attempt to be concise and amusing we were unclear. Dr. McIsaac is correct that we conflated the values of the parameter $\theta$ with the validity of the reports. To clarify, consider this revised example.

Say we have chosen at random one of two unfair coins which are weighted so that coin one and coin two land heads on average $1 / 3$ and $2 / 3$ of throws, respectively. Rather than consulting experts, we flip the chosen coin 12 times and it lands heads 7 times. The results in our Table hold using this revised example.

Dr. McIsaac introduced a parameter $\pi$ to index the validity of the data reports and thereby separate the data validity from the value of the parameter of interest $\theta$. Here we briefly offer another perspective to distinguish the value of the parameter of interest $\theta$ from the validity of the data. To extend our revised example, say we were not able to flip the coin but instead gathered reports from 12 others who did flip the coin. Say the data are unchanged: 7 heads in 12 throws reported. If we were to assume sensitivity and specificity of 1 , then the results in our Table again hold. However, imagine we had prior knowledge or validation data suggesting that the sensitivity and specificity of a heads report were exactly 0.7 and 0.8 , respectively. Then the modified likelihood at $\theta=2 / 3$ is 0.21515 and the posterior probability of $\theta=2 / 3$ is reduced from 0.8 under perfect sensitivity and specificity to 0.75 , as might be expected. This modified likelihood is $\left(\begin{array}{l}n \\ y\end{array}\right)\{s e \theta+(1-s p)(1-\theta)\}^{y}\{s p(1-\theta)+(1-s e) \theta\}^{(n-y)}$, and allows for imperfect sensitivity (se) and specificity (sp) of reports yet collapses to the standard form ${ }^{1}$ when sensitivity and specificity are both one. 
Generally, one should strive to judge the validity of data separately from the data values. But in epidemiology, as in life, the rabits (random bits) of information received are partially divorced from context. Of course, this dialog itself simply represents a working-model or useful fiction, which may be absurd in particular contexts. If we think the data are not at all associated with reality then we will view all information as noise not signal. Such radical skepticism is a form of dogmatism. Nihilists cannot learn. On the other hand, the likelihoods we and Dr. McIsaac presented assume that we are equally certain about results from all sources. If, in an attempt to distinguish alternative facts from reality, we assign different values of sensitivity and specificity for different reports, then there is a danger in selectively reinforcing our priors, driving ourselves towards dogma, even if we begin with an open mind.

In this après-truth world, 3 , page 159 it may be helpful to state conclusions explicitly. We reaffirm our initial claim that dogmatists cannot learn, ${ }^{2}$ with Dr. McIsaac's appendix that non-dogmatists can be led astray by incorrect information, in the forms of mistaken prior or imperfect data.

\section{References}

1. McIsaac M. Re. Dogmatists Cannot Learn. Epidemiology. 2017; 28:xxx.

2. Cole SR, Chu H, Brookhart MA, Edwards JK. Dogmatists Cannot Learn. Epidemiology. 2017; 28(2):e10-e11. [PubMed: 27787291]

3. Blackburn, S. Truth. New York: Oxford; 2005. 